\title{
Pilot study: Decreasing parental anxiety during peanut oral immunotherapy
}

\author{
Kinga Olson*1, Ruth Rosenblum² \\ ${ }^{1}$ California State University Fresno, Fresno, CA, USA \\ ${ }^{2}$ The Valley Foundation School of Nursing, San Jose State University, San Jose, CA, USA
}

Received: October 7, 2019

DOI: $10.5430 /$ jnep.v10n3p65
Accepted: November 4, 2019

Online Published: November 25, 2019

\begin{abstract}
Objective: Peanut allergies are common in the pediatric population. Peanut oral immunotherapy (OIT) can cause anxiety for children and families. A pilot study was conducted to determine what elements parents consider most useful in reducing anxiety within a newly implemented OIT program.

Methods: A convenience sample of parents $(n=15)$ was surveyed to measure perceptions of specific anxiety-reducing elements at a private allergy practice.

Results: The 10-question parent survey utilized a Likert Scale measuring how various elements of the OIT program reduced their anxiety. All elements that were provided directly by the clinic received favorable ratings.

Discussion and conclusions: Commercially prepared peanut OIT will soon receive FDA approval, and allergy clinics will consider implementing this new therapy for peanut allergic patients. Food allergies invariably cause anxiety for parents and children, therefore careful consideration of how to decrease anxiety during OIT therapy was examined in this pilot study.
\end{abstract}

Key Words: Peanut Oral Immunotherapy, Quality of life, Food allergy anxiety

\section{INTRODUCTION}

The privilege of providing a therapy perceived by patients and families as "life changing" has seasoned healthcare providers excited about the evidence-based research involving food oral immunotherapy. ${ }^{[1]}$ Living with food allergies can be distressing due to the daily fear of exposures and anaphylaxis. Decreased quality of life for food allergic children and their families has prompted numerous immunotherapy research projects over the past two decades. ${ }^{[2-6]}$ Since parental anxiety commonly accompanies food allergies, means of reducing anxiety during OIT warrants consideration.

Peanut allergies affect 1 in 70 children in the United States (US), are considered one of the most prevalent food aller- gies, ${ }^{[3]}$ and are responsible for over half of all food-related anaphylactic deaths in the US. ${ }^{[7]}$ Peanut allergies most commonly begin in childhood, and $80 \%$ do not outgrow them. ${ }^{[8]}$ Stress and anxiety on the child and parents of children with peanut allergies may be overwhelming due to the daily threat of accidental ingestions. In the past, avoidance has been the only option, and clearly not always achievable since as many as $50 \%$ of peanut allergic individuals have reported accidental ingestion over a 2-year period. ${ }^{[3]}$ This portrays how minimal exposures can cause life-threatening reactions, as is the case with cross-contaminated foods. Peanut allergy research has developed promising new technologies ${ }^{[3]}$ over the past decade and today many clinicians are using this research to introduce peanut OIT to patients and families

*Correspondence: Kinga Olson; Email: kingaolsonFNP@gmail.com; Address: 1501 Santa Inez Dr, San Jose, California, USA. 
looking for another option.

OIT is a medical treatment that allows the immune system to become desensitized to a food to which it may otherwise be allergic. This is not a cure for food allergies but a way to decrease the incidence of anaphylaxis due to accidental ingestion. Most recently, two biopharmaceutical companies (Aimmune Pharmaceuticals and Viaskin Peanut Technologies) have received Fast Track and Breakthrough Therapy designation from the US Food and Drug Administration (FDA), promising approved peanut desensitization therapy products commercially available as early as 2019. ${ }^{[9]}$ Once these therapies receive FDA approval, it is anticipated that more healthcare providers and institutions will be considering implementation.

Numerous peer-reviewed publications have discussed various peanut OIT study protocols, maintenance dosing, safety concerns, as well as the overwhelming evidence that peanut OIT improves quality of life for the recipients and their families. $^{[2-6]}$ It is imperative that clinicians considering this therapy for their patients understand that most people and families living with food allergies are also very anxious about experiencing anaphylaxis. Currently there are no published studies discussing decreasing parental anxiety during OIT therapy, nor any discussions about what elements of a program are viewed as most valuable by parents. Querying parents about the specific anxiety-reducing facets of a program could be a valuable outcome measure for a program evaluation.

\section{Review of Literature}

The daily threat of anaphylaxis causes anxiety for food allergic (FA) children and caregivers, ${ }^{[10]}$ and for many it defines their lifestyle. Parents feel a daily threat of their child potentially having a life-threatening reaction each time they eat. The anxiety that develops from this fear can also lead to overprotective behaviors by restricting a child's diet, playdates, social events, and travel; children may also develop learned behaviors such as helplessness when parents control every aspect of their child's life. ${ }^{[11]}$ A third of FA children report being bullied for their food allergies, ${ }^{[12]}$ and according to Polk \& Dinakar, ${ }^{[13]}$ peanut allergic children experience greater anxiety when eating at social events than children with insulin-dependent diabetes. These and other similar experiences significantly affect quality of life in children and caregivers.

An alternative therapy for treating food allergies is oral immunotherapy. ${ }^{[2]}$ As this treatment becomes more available to healthcare institutions, managing FA anxiety may become a key element of any OIT program, as evidenced by a study focusing on the quality of life (QOL) of these patients and their families before, during, and after OIT. ${ }^{[14]}$ The authors report that QOL temporarily decreases during the process of OIT for some families, but improves significantly once maintenance dosing (or even partial desensitization) is achieved. This temporary decrease of QOL during OIT therapy warrants further discussion, including how to minimize anxiety during therapy.

Creating a survey that assesses anxiety-reducing components may aid in future OIT program development. Herbert, Shamesh, and Bender's 2016 ${ }^{[15]}$ clinical management review article about FA anxiety described how families reported benefit from having their medical provider listen to their concerns while discussing food allergies. Behavioral interventions addressing parent's needs of children with food allergies are few, but are touched upon in this report suggesting parent support groups and workshops improving food allergy competence and decreased burden. Other valuable findings of this review included parental anxiety and psychosocial concerns related to food allergies and parents admitting that they struggle to recognize symptoms of mild food allergy symptoms versus anaphylaxis (2016). Findings from Herbert et al.'s article ${ }^{[16]}$ are especially useful during educational protocol development for any OIT program, and was especially helpful during creation of the parental survey used in this program evaluation.

Epstein-Rigbi et al. ${ }^{[14]}$ focus on how QOL is affected throughout the OIT process. This prospective cohort study utilized the Food Allergy Quality of Life QuestionnaireParent Form (FAQLQ-PF), a validated testing tool developed to examine the QOL of children as assessed by their parents. The results report a consistent improvement in QOL for these families once the patient reaches maintenance dosing. This article points out, however, that additional anxiety is experienced by these families during the OIT process itself, concluding that implementing methods to overcome these challenges are beneficial for the overall experience.

Investigating Lerwick's four treatment principles for minimizing anxiety during healthcare visits and therapies choices, agenda, resilience, emotional support (CARE) provides useful information during OIT program development. ${ }^{[16]}$ Expanding on these treatment principles offer further understanding of the importance of CARE such as offering choices about their care, offering an agenda (or schedule) makes the healthcare experience clear from the start, reducing the fear of the unknown. Discovering patient and parent resilience and discussing emotions are important for healthcare providers to explore with their patients in order to minimize anxiety. 


\section{MethodS}

\subsection{Project design}

A pilot study survey was conducted six months after implementation of a new peanut oral immunotherapy program, focusing on parental anxiety reduction. Lerwick's CARE principles were considered during the peanut OIT program development so as to include elements in the program that would help reduce parental anxiety before and during therapy. The importance of these elements were tested by incorporating parental input of patients enrolled in the program via survey. This survey was created to measure parent's perception of how specific elements of the OIT program helped reduce anxiety during the OIT experience, using a ten question survey which rated anxiety-reducing elements using a Likert scale (5=strongly agree, $1=$ strongly disagree).

\subsection{Setting and Sample}

The new implemented peanut OIT program was offered to all established patients of a private allergy clinic in Silicon Valley. Clinic providers include 8 physicians, 3 nurse practitioners, and 3 dedicated OIT-trained nurses. The allergy clinic has a diverse population of patients and providers (including Caucasian, Vietnamese, Chinese, Korean, Thai, Indian, Filipino, Hispanic, and African-American), with payers consisting of a mix of private insurance, Medicaid, Medicare, and self-pay patients. Participant size was small $(n=15)$ since this was a pilot study consisting of parents of children undergoing peanut OIT. The average age of the patient population was 8 years old (ranging in age $4-15$ years). The 15 parents (12 mothers and 3 fathers) made up the evaluation sample and was representative of the community as described earlier. Criteria for inclusion for this program evaluation included all parents of children participating in OIT therapy. Exclusion criteria included parents that chose not to provide informed consent or did not participate in the survey. All parents that were enrolled in the clinic's peanut OIT program volunteered to participate in the program survey during the second half of their child's desensitization program (after 12 weeks of starting OIT). No parents declined taking the survey.

\subsection{Program Design and Implementation}

During the peanut OIT program design phase, a taskforce was created consisting of "champion" physicians and nurse practitioners which were responsible for gathering evidence for implementing peanut OIT in a "non-institutional" setting. After critiquing and synthesizing the validity and appropriateness of the evidence, guidelines were created with regard to protocols, safety, dosing, and maintenance targets, utilizing the published research. A 26-week OIT program was designed where the patient comes to the allergy clinic every Published by Sciedu Press two weeks for an escalation dose of peanut protein, followed by daily dosing at home. The providers also established mentoring relationships with other allergy clinics with OIT programs, receiving valuable advice in regards to the logistical aspects of program development.

Providers of patients with FA understand the complexities of anxiety that shadow a diagnosis of peanut-related anaphylaxis, thus incorporating anxiety-reducing elements within this type of program became a priority for everyone involved. Using Lerwick's CARE principles for reducing anxiety during healthcare therapy, the nurse practitioners of the clinic developed practices for reducing parental anxiety during the peanut OIT program, as noted in Table 1. Each of the defined practices were thoroughly described to patient families prior to enrolling in the peanut OIT program, thus all families were aware of available resources, even if they did not use them.

\subsection{Measures}

The OIT pilot study survey included measures involving parental anxiety with OIT therapy by utilizing an IRBapproved ten-question survey listing anxiety-reducing elements the providers considered useful for any OIT program (internal consistency was measured informally by comparing answers and noting consistency with results between surveys; reliability was determined via expert review). The survey was given to each patient's parent during the second half of their OIT therapy (after completing 12 weeks of therapy) and took place from September 2018 through February 2019. Parents were asked to rate how helpful each element of their OIT experience was in decreasing their anxiety $(5=$ most helpful, $1=$ least helpful). Before completing the survey, the parents signed a consent form that explained confidentiality and anonymity of the completed questionnaires. Additional comments could be given by the parents in an open-ended question at the end of the survey reading: "Please offer any further suggestions on how to decrease anxiety during the desensitization program".

\subsection{Data collection}

Precautions taken to minimize risks included confidentiality and anonymity of parental evaluations: Since receiving honest parental survey answers was important, parents were informed that the surveys are kept anonymous and this was ensured by instructing parents to omit names from the survey. The survey was administered by one of the OIT nurses, no names of patient, parent, or associated provider were on the surveys and the completed surveys were placed in blank individual envelopes that the parent could seal upon completion. To insure maximum number of returned surveys, parents 
were given the survey during their child's dose escalation appointment, where they are required to wait 60 minutes post dose escalation for observation of adverse reactions. Completing the surveys were purely voluntary, and no com- pensation was provided for the parents participating in this evaluation. All 15 parents of the program sample agreed to complete the survey achieving $100 \%$ return.

Table 1. Proposed anxiety reducing elements for OIT program
1. An hour long orientation program for interested patients and families to introduce the science of peanut OIT, and basic instructions that will be included in the program.
2. An orientation folder provided during the orientation program including answers to frequently asked questions, including how to manage allergic reactions, a time table for therapy, and provider contact information.
3. An administrator providing information in regards to financial obligations, co-pays, and insurance reimbursement for the program, in order to decrease the anxiety of unexpected healthcare expenses.
4. Establishing OIT- trained providers for continuity of care during OIT therapy visits.
5. Providing 24/7 advice to patients was previously established by using a call service that pages the on-call clinic providers during off hours. This feature became especially important for OIT patients and parents in order to reach a provider during an emergency. Offering email contact for less emergent questions was managed by the lead OIT nurse practitioner.
6. Prior to referring a patient to the peanut OIT program, physician providers were encouraged to have a thorough discussion about the pros and cons of OIT with parents, thus establishing long term goals and trust in the program.
7. The nurse practitioner gave detailed education about common occurrences during OIT in order to empower the patient/parent with useful references throughout the program. The first educational session lasting approximately 15-30 min, takes place during the initial OIT clinic visit.
8. Established a professional relationship with a local psychologist that understands how to treat food allergic anxiety in case referrals are warranted.
9. Provided “quiet” activities during escalation appointments in order to keep young children from becoming physically active during the hour long observance period.
10. Had "experienced" OIT parents available to speak with anxious parents still considering if OIT is right for their child or not.

\subsection{Study analysis}

Parents were asked to complete the peanut OIT program survey to assess what elements of the OIT experience were found to be valuable in decreasing their food allergy-related anxiety. The ten questions measured activities that helped ease a parents anxiety about the food allergy program. The answers were assigned a Likert scale value in order to calculate mean scores for each question as noted in Survey Results documented in Appendix.

\section{Results}

\subsection{Primary outcomes}

Survey results were compiled (see Appendix) with the following scores: Education provided by their allergist is considered very helpful (score mean $=4.7$ ) in easing parental anxiety. Attending an hour long orientation meeting discussing how OIT works and the commitment that is involved also scored high in reducing parental anxiety (mean $=4.8$ ). The parent's take-home orientation folder explaining OIT, timelines, how to treat adverse symptoms, and contact information also received high scores in easing anxiety (mean $=$ 4.7).

The first phase of OIT includes the initial escalation day that takes approximately 5 hours and involves a series of very small doses increasing in peanut amount spaced 30 minutes apart referred to as oral challenges (OC). After the OC is completed, the OIT providers have the opportunity to educate the patients and parents in more detail about how to treat a variety of symptoms at home (allergic versus viral illness, etc.). Patients and parents are also encouraged to practice using the epinephrine injectables as part of the education provided. Completing this education scored high among the parents in easing anxiety, resulting in a 4.7 rating. Having an allergist provider available to speak to the parents 24 hours a day, 7 days a week also rated high with a mean score of 4.7 in easing parental anxiety. Since food allergy anxiety can be serious, allergists consulting with psychologists specializing in food allergy anxiety could be seen as beneficial, and was a priority for the pilot study providers. None of the parents or subjects in this program evaluation warranted or requested referral to counseling, but having the option available proved value in decreasing parental anxiety with a rating of 4.3. Having quiet diversional activities available during the lengthy appointments were also rated 4.1 in easing anxiety, however most families brought their own activities and electronic devices for entertainment. Parents today are more apt to initiate their own research about food allergies 
and OIT, and those that did or spoke to friends about OIT rated these exercises 4.3 and 4.1 respectively in easing anxiety. Interestingly enough, online searches and blogging on food allergy websites only scored an average of 3.5 in easing parental food allergy anxiety. Additional comments given by the parents in an open-ended question at the end of the survey read: "Please offer any further suggestions on how to decrease anxiety during the desensitization program" and are included in Table 2 of Survey Responses.

Table 2. Open-ended survey responses

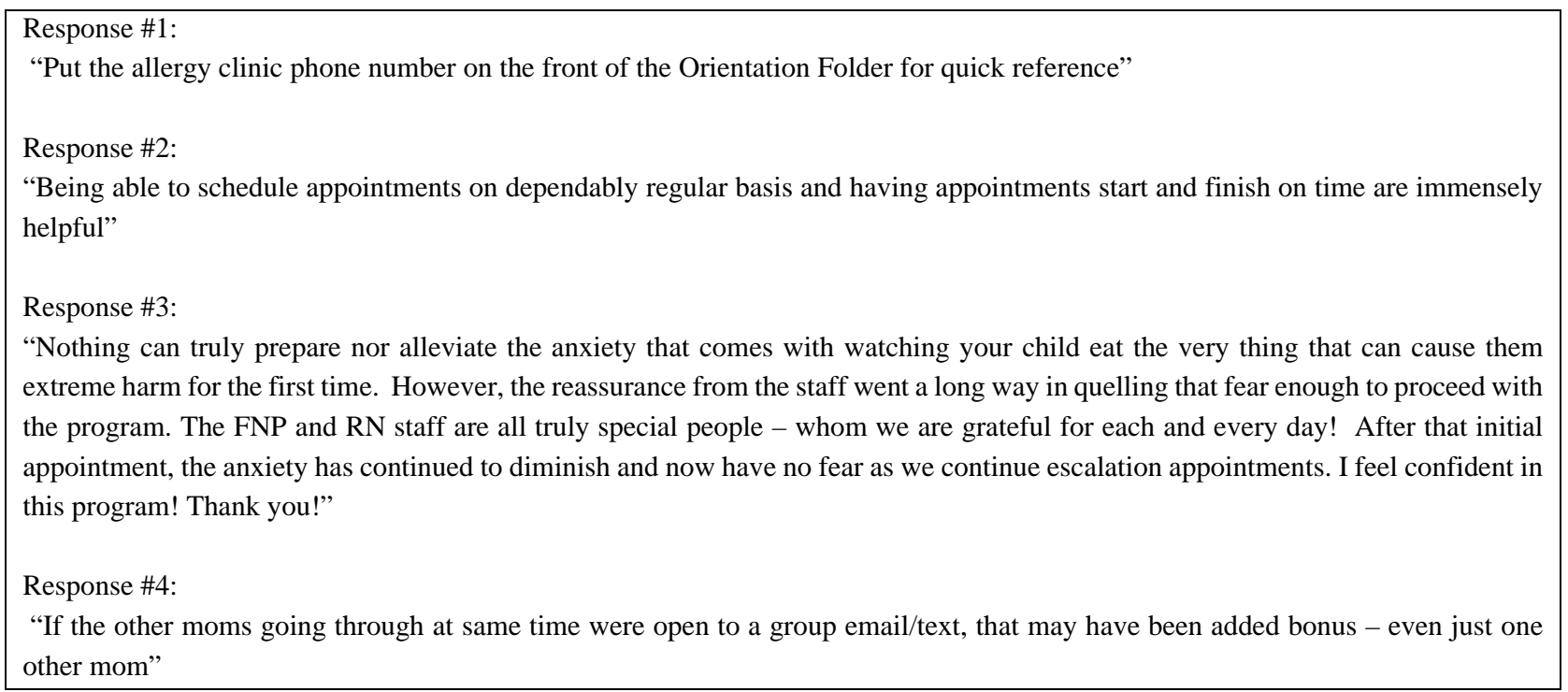

\section{Discussion}

Food allergy awareness has become such a public health concern that Healthy People 2020 created goals to aid in the reduction of food allergy-related anaphylaxis. ${ }^{[17]}$ Peanut OIT is one way to help reduce food allergy-related anaphylaxis, and to be successful in implementing this, addressing food allergy anxiety must be part of the solution. Including a parentcompleted survey within a pilot program evaluation provided valuable insight to this pilot study by evaluating how particular aspects of an OIT program helped ease parental anxiety. One key point that was established by the survey included the importance of parental education before and during the OIT program. Providing an orientation hour with the parents before starting a peanut OIT program teaches them about how OIT works, how their child may react, and how the provider will help them manage the next 6 months. It is crucial that parents understand the daily commitment involved, and the protocols that are required for their child's safety. Having all this information provided in an informational guide book allows the parent to go home and process the information before making the commitment. This pilot study found that approximately $25 \%$ of the families that attended orientation decided that they were either not ready or postpone starting OIT until they had more time (such as summer months). As a result however, $100 \%$ of families that enrolled into the program were fully committed, followed the protocols carefully, and graduated successfully from the program.

While providers discuss OIT as an optional therapy with their patients, it is important to include the risks and benefits of OIT for clear understanding of the therapy, thus providers need to stay current with food allergy research since breakthrough studies continue to be published. Program safety discussions with the families have been found to be the cornerstone to the success of this program, and were valued by parents with high survey ratings. The best opportunity for this occurs on day-1 of initial therapy. During this time the patient remains in the office for additional observational time, providing time for more detailed education to occur for parents. This additional education includes treating mild, moderate, severe symptoms (including epinephrine injectable demonstration and practice), and what to do if the child is sick with viral illness, travels to higher elevations, or misses a dose. All this is documented and kept in the patient's personal OIT diary for further reference, thus empowering the parents with their own detailed information. Having this individualized education period at the end of the first day works well since the anxiety levels tend to decrease once the initial escalation dosing is complete, and parents are more focused on the education that is being provided.

Parents are looking for a quality of life improvement with OIT, yet they fear harm will come to their child. Therapy 
provided by an allergist team they have developed a relationship with while being treated for various environmental allergies, asthma, immunology disorders, and/or food allergy management, gives the entrusted providers an advantage when adding peanut OIT to their services. Since increasing the patient's peanut tolerance occurs in a safe clinic environment, parents can be confident that emergency treatment can be provided immediately if needed. When a parent has a question about their child's OIT therapy, (especially one that warrants immediate attention), providing 24/7 on-call provider coverage is also invaluable and appreciated. Interestingly, OIT parents rarely called for assistance during the pilot study, speculating that since they were provided with a guide in their orientation book, this empowers parents and patients to take care of minor/moderate/severe allergic reactions themselves. Secure electronic mail was also found to be a convenient means of communication when parents had less timely information they need to discuss (such as scheduling conflicts, upcoming travel plans, etc.).

Having a working relationship with a licensed psychologist specializing in food allergy anxiety can also be beneficial when referring any parents requiring more specialized counseling. Parents at shared escalation appointment times were able to connect and create their own "parent support group" where they were able to discuss various topics while their children were being observed after dosing. Creating an environment which facilitates this type of community within the clinic would benefit many parents and children, (keeping confidentiality issues in mind).

Limitations of this pilot study evaluation includes the small sample size, with 15 parents and 15 children. Since this program evaluation used a convenience sample, no blind or control groups were applied. Parental roles (mothers versus fathers) may have led to survey response bias, and in the future, surveying both parents would be recommended. Another limitation may be that the sample came from a geographically non-diverse group of parents living in the Silicon Valley.

\section{Implications}

Despite reported adverse reactions being common in OIT (mild skin or mouth itching, or abdominal discomfort), the ability to receive this therapy has become very desirable by many parents since the margin of safety for FA individuals is greatly improved, and safety is an essential concept for patients, parents, and clinicians. ${ }^{[18]}$ However, the process of undergoing OIT with their children causes much parental anxiety, especially when they are asking their peanut allergic child to eat the very food that can cause great harm. ${ }^{[15]}$ Utilizing Lerwick's CARE principles for reducing anxiety during healthcare therapy directed nurse practitioners to implement a variety of anxiety reducing practices in the peanut OIT pilot program. The parent survey results reported favorably in regards to the CARE principles that were implemented.

Though sample size may be seen as a limitation to this study, initiating the discussion of how OIT programs will address parental anxiety is crucial. Future studies should also include age-appropriate elements of a program that helps reduce anxiety for children undergoing OIT as well.

\section{Conclusions}

Peanut OIT is recognized as an optional therapy that decreases the incidence of anaphylaxis due to accidental peanut ingestion by introducing small amounts of peanut powder. ${ }^{[18]}$ Peanut OIT has also shown to improve QOL for peanut allergic individuals and their families. ${ }^{[14]}$ Care providers eagerly anticipate evidence-based practice guidelines to be published once FDA approves new peanut immunotherapy pharmaceutical products. This is the first program evaluation with survey that takes into consideration parental anxiety reducing elements of a peanut OIT program, pointing to key strategies for minimizing food allergy related anxiety for parents. These strategies include developing and implementing educational programs within OIT to help prepare patients and their families with knowledge about the process and what to expect during therapy, and how to treat allergy related symptoms, including anaphylaxis. An effective means of communications between providers and patients is also encouraged since provider availability is seen as an anxiety-reducing element of therapy by this evaluation.

\section{CONFliCts OF InTEREST Disclosure}

The authors declare that they have no competing interests.

\section{REFERENCES}

[1] Wasserman R, Jones D, Windom H. Oral immunotherapy for food allergy, The FAST perspective. Annals of Allergy, Asthma and Immunology. 2018 121: 272-275. PMid:29928983 https://doi.or $\mathrm{g} / 10.1016 / \mathrm{j}$. anai.2018.06.011
[2] Pajno G, Fernandez-Rivas M, Arasi S, et al. EAACI Guidelines on allergen immunotherapy: IgE mediated food allergy. Allergy European Journal of Allergy and Clinical Immunology. 2017; 1(17).

[3] Bird J, Spergel J, Jones S, et al. Efficacy and safety of AR101 in oral immunotherapy for peanut allergy: Results of ARC001, a random- 
ized, double-blind, placebo-controlled phase 2 clinical trial. Journal of Allergy and Clinical Immunology Practice. 2018; 6(2).

[4] Wasserman R, Factor J, Baker J, et al. Oral immunotherapy for peanut allergy: Multipractice experience with epinephrine treated reactions. The Journal of Allergy and Clinical Immunology: In Practice. 2014; 2(1). PMid:24565775 https://doi .org/10.1016/j . jaip. 201 3.10 .001

[5] Wasserman R, Hague A, Pence D, et al. Real-world experience with peanut oral immunotherapy: Lessons learned from 270 patients. Journal of Allergy and Clinical Immunological Practice. 2019; 7(2). PMid:29859333 https://doi.org/10.1016/j.jaip. 2018.05 .023

[6] Bégin P, Chinthrajah R, Nadeau K. Oral immunotherapy for the treatment of food allergy. Human Vaccines \& Immunotherapeutics. 2014; 10(8): 2295-2302. PMid:25424935 https ://doi .org/10.4161/ hv. 29233

[7] Wood R. Oral immunotherapy for food allergy. Journal of Investigative Allergology and Clinical Immunology. 2017; 27(3). PMid:28102823 https://doi.org/10.18176/jiaci.0143

[8] FARE. Peanut allergy: learn about peanut allergy, how to read food labels and how to avoid eating peanuts [Internet]. McLean, VA: Food Allergy Research \& Education. [cited 2018 Nov 20]. Available from: https://www.foodallergy.org/research-programs/ research-grants

[9] Tilles S, Petroni D. FDA-approved peanut allergy treatment, The first wave is about to cress. Annual Allergy, Asthma, and Immunology. 2018; 121: 145-149. PMid:29909055 https://doi.org/10.101 $6 / j$.anai.2018.06.005

[10] Broome S, Lutz B, Cook C. Becoming the parent of a child with life-threatening food allergies. Journal of Pediatric Nursing. 2015; 30: 532-542. PMid:25458106 https://doi.org/10.1016/j.pe dn. 2014.10 .012
[11] Quach L, John R. Psychosocial impact of growing up with food allergies. The Journal for Nurse Practitioners. 2018; 14(6). https: //doi.org/10.1016/j.nurpra.2018.03.017

[12] Peck J, Larson K. One child's food fight: A case study of oral immunotherapy treatment for food allergies. Journal of Pediatric Health Care. 2018; 32(4). PMid:29540279 https://doi.org/10.1016/ j. pedhc. 2018.01.004

[13] Polk B, Dinakar C. Patient-centered outcomes in food allergy. Food Allergy. [cited 2017 Nov 20] Available from: https ://www . ncbi .nlm.nih.gov/pubmed/28516366

[14] Epstien-Rigbi N, Goldberg M, Levy M, et al. Quality of life of food-allergic patients before, during, and after oral immunotherapy. Journal of Allergy and Clinical Immunology Practice. 2019; 7(2). PMid:30129441 https://doi.org/10.1016/j.jaip.2018.06 .016

[15] Herbert L, Shemesh E, Bender B. Clinical management of psychosocial concerns related to food allergy. Journal of Allergy Clinical Immunology Practice. 2016; 4(4). https://doi.org/10.1016/j. jaip.2015.11.006

[16] Lerwick J. Minimizing pediatric healthcare-induced anxiety and trauma. World Journal of Clinical Pediatrics. 2016; 5(2): 143-150. PMid:27170924 https://doi.org/10.5409/wjcp.v5.i2.143

[17] Healthy People 2020: Food Safety FS-4, Reduce severe allergic reactions to food among adults with a food allergy diagnosis. Rockville, MD: Office of Disease Prevention and Health Promotion (cited 2018 Nov 20). Available from: https://www.healthypeople.gov/2020/topics-obj ectives/topic/food-safety/objectives\#4495

[18] Shreffler W, Baumert J, Remington B, et al. The importance of reducing risk in peanut allergy: Current and future therapies. American College of Allergy, Asthma \& Immunology. [cited 2018 Oct 15] PMid:29289463 https://doi.org/10.1016/j.anai.2017.10 .038 